\section{Women's Health across the Lifespan: A Pharmacotherapeutic Approach}

Borgelt LM, O'Connell MB, Smith JA, Calis KA, editors. American Society of Health-System Pharmacists, Bethesda, Maryland, 2010. 864 pages. Softcover. ISBN 978-1-58528-194-7. US\$114 (US\$104 for ASHP members).

Women's Health across the Lifespan: A Pharmacotherapeutic Approach was developed as a comprehensive resource on the health care needs of women throughout their life stages. The book also addresses the influence of sex on pharmacokinetics and response to drug therapy. Broader issues affecting health are also discussed, such as psychosocial, ethical, and racial issues.

The book is organized into 8 sections: historical and social context; biology and physiology; menstrual health; contraception; preconception, pregnancy, and postpartum care; chronic illnesses that affect women differently than they affect men; cancers; and ethical issues. Each chapter has a standard template, covering epidemiology, clinical presentation, pathophysiology, guidelines, and treatment. Information on complementary and alternative medicine is interspersed throughout the book, where appropriate.

The chapters are well organized and easy to follow, with clear headings. Objectives are presented at the beginning of each chapter to help readers assess their learning. Each chapter also includes a case to highlight concepts in practice, with background information appearing at the beginning of the chapter, and the assessment and treatment plan at the end. These cases are practical tools for applying the chapter content. The various authors also make good use of tables and flow charts to present important content. Additional information, such as other tables, figures, and practice cases, are available online.

The "Therapeutic Challenge" questions represent another helpful feature of this book. Each of these questions addresses a controversial issue in women's health, and the questions are intended to spur discussion or investigation into the topic. They could be used as teaching strategies to promote critical thinking to students, residents, or new practitioners. Unfortunately, there is no discussion related to the answers to these Therapeutic Challenge questions. Advanced practitioners using the book as a quick reference to help with more complex problems will need to access other resources for this information.
The nearly 120 contributors include a range of pharmacists, physicians, and other health care professionals. The chapter content is well referenced. However, because of space limitations, the references are not included in the print version but rather are available online. Some readers may find this inconvenient; however, it has benefits because references are hyperlinked to PubMed citations when possible.

This is one of the most comprehensive books I have seen on the topic of women's health. With its pharmacotherapeutic approach, it is definitely targeted to pharmacists; however, it would be a useful resource for any health care professional. The book is written for practitioners in the United States, as reflected in the primarily US brands and the use of non-SI units, but the therapeutic content is applicable to Canada.

For the most part the chapters are very thorough. Unfortunately, there was some inconsistency among chapters, especially with regard to discussion of guidelines. For example, the menopause chapter makes little mention of position statements from organizations such as the North American Menopause Society, whereas other chapters have more discussion of guidelines. In addition, I found that some of the tables were missing information. For example, the table of hormone therapy products does not list micronized progesterone, although this product is discussed in the text.

In summary, this book is a unique compilation of issues relevant to the whole continuum of women's health. The editors should be applauded for their holistic approach, which goes beyond pathophysiology to consider other factors that influence women's health, such as culture, gender differences, environmental factors, and health care access. Pharmacists practising in women's health will find this book an excellent reference, especially for a quick review of topics beyond their area of expertise or for teaching purposes.

Nese Yuksel, BBScPharm, PharmD, ACPR, FCSHP, NCMP

Faculty of Pharmacy and Pharmaceutical Sciences

University of Alberta

Edmonton, Alberta 